\title{
DESAGREGANDO LOS QUALIA: UN ANÁLISIS DE SU FUNCIÓN EN LOS PROCESOS COGNITIVOS
}

\author{
Rodolfo BÄChler Silva* \\ doi:10.11144/Javeriana.uph35-70.dqpc
}

\begin{abstract}
RESUMEN
Se expone una reflexión acerca de los distintos tipos de propiedades fenomenológicas de algunos estados mentales, también conocidos como qualia, con el objetivo de identificar los roles que cumplen en la cognición. Para ello se releva primero el carácter cualitativo de la fenomenología, proponiéndose que conocer es un proceso que consiste en dar nuevos formatos a la experiencia a través de su progresiva explicitación. En segundo lugar, se analizan las características de dos diferentes tipos de qualia y sus papeles en la cognición. Un primer tipo, de características intrinsecamente cognitivas, se encuentra asociado a las emociones y otros estados similares que participan como centro del pensamiento, producto de su posibilidad de acceso a la autoconsciencia. Un segundo tipo, conformado por qualia vinculados a la percepción, corresponde a fenomenología autoconscientemente inaccesible, razón por la cual juega un rol representacional que no depende de sus características cualitativas específicas.
\end{abstract}

Palabras clave: qualia; emoción; sensación; conocimiento; experiencia

* Núcleo Psicología, Facultad de Ciencias, Universidad Mayor, Chile.

Correo electrónico: rodolfo.bachler@umayor.cl

Para citar este artículo: Bächler Silva, R. (2018). Desagregando los qualia: un análisis de su función en los procesos cognitivos. Universitas Philosophica, 35(70), pp. 15-41. ISSN 0120-5323, ISSN en línea 2346-2426 doi:10.11144/Javeriana.uph35-70.dqpc 


\title{
DISAGGREGATING QUALIA: AN ANALYSIS OF THEIR FUNCTION IN COGNITIVE PROCESSES
}

\author{
Rodolfo BäCHLER SiLVA
}

\begin{abstract}
This article presents a reflection about the different types of phenomenological properties of mental states, also known as qualia. With the objective of identifying the role of qualia in cognition, the qualitative nature of phenomenology is discussed. It is proposed that knowing is a process by which new forms are given to experience through the progressive elucidation of its qualitative properties. Second, the characteristics of two different types of qualia and their roles in cognition are analyzed. A first type, with intrinsically cognitive characteristics, is associated with emotions and other similar states that participate as a center of thought, as a result of their accessibility to self-consciousness. A second type, consisting of perceptual qualia or sensations, corresponds to consciously inaccessible phenomena, and thus plays a representational role that does not depend on its specific qualitative characteristics.
\end{abstract}

Keywords: qualia; emotion; sensation; knowledge; experience 
TANTO El COGNitivismo, QUe CONSIDERA LA COGNición como manipulación de símbolos, como el paradigma corporalizado de la mente, que destaca el rol del cuerpo como soporte estructural y sensorio-motriz de los procesos cognitivos, son enfoques que dejan el componente cualitativo de la cognición fuera del estudio científico de la mente. Hablamos de la explicación de los qualia, un concepto que denota la existencia de propiedades cualitativas asociadas a diferentes tipos de estados mentales tales como el dolor, las sensaciones, las emociones $^{1} \mathrm{y}$, en general, una extensa variedad de fenomenología cuya investigación ha sido relegada al campo de la reflexión filosófica, principalmente. Desde este terreno se ha avanzado en determinar algunas de las propiedades que poseen dichos estados y que suponen obstáculos para su investigación científica, pero no se explica cuál es el rol que juegan en el funcionamiento global de la mente y la cognición. Lo que desde este enfoque se busca es la comprensión de las causas y modos que determinan su surgimiento y configuración y, especialmente, la explicación de su relación con el mundo físico. Sobre este tema en particular, existen variadas perspectivas que no analizaré en este trabajo, pero que, dicho sucintamente, varían desde aquellos enfoques que de diferentes formas niegan la existencia de este tipo de fenómenos (Churchland, 1981; Dennett, 1995), hasta algunas teorías de orden más fenomenológico, que destacan la experiencia cualitativa como una característica fundamental de la mente (Flanagan, 1991; Searle, 1996; Varela, 2000). En medio, se encuentran quienes piensan que los qualia constituyen un misterio, es decir, un problema para el cual no existe solución con las herramientas cognitivas de las cuales disponemos (McGinn, 1989; Nagel, 1974).

No obstante, si asumimos la existencia de los qualia más allá de explicar su relación con el mundo físico, vale la pena preguntarse si dichas propiedades juegan algún papel en el funcionamiento cognitivo y cuál sería. Por ejemplo, ¿existen diferencias para aquel proceso cognitivo que llamamos reflexión que se deriven de

1 Aunque la literatura no suele hacer referencia a qualia emocionales, tomándose generalmente como casos prototípicos de este tipo de propiedades el dolor o las sensaciones perceptivas, existen autores que identifican propiedades cualitativas de las emociones tales como DeLancey (1996), quien argumenta, además, que los qualia de la experiencia emocional proveen un ejemplo que resulta crítico para poder especular filosóficamente sobre el rol funcional de los estados fenomenológicos conscientes. 
la presencia de diferentes qualia emocionales mientras este proceso cognitivo se realiza? Y respecto de la presencia de qualia asociados a la percepción: ¿varía de algún modo el proceso cognitivo que llamamos percepción cuando este se realiza acompañado de diferentes sensaciones o qualia perceptuales? ¿Qué consecuencia tendría en términos cognitivos si, por ejemplo, cada vez que nos enfrentamos a la percepción visual del pasto, en vez de constituirse una sensación de verdor en el espacio mental interno, ocurriera que experimentamos rojez u otra experiencia de color, o incluso, una sensación de tipo auditivo o kinestésico? ¿Resulta modificada nuestra percepción en sus aspectos cognitivos si se produce una variación de sus propiedades cualitativas asociadas? En síntesis: ¿qué función cumplen las propiedades cualitativas de los estados mentales respecto de aquellos procesos que solemos identificar como cognitivos, tales como pensar, percibir, etc.?

Para analizar adecuadamente el rol de la fenomenología en el funcionamiento global de la mente y responder preguntas como las anteriores, se requiere dar dos pasos previos que nos permitan clarificar con mayor exactitud de qué estamos hablando cuando decimos qualia. Se trata de un problema que es imprescindible despejar debido, entre otras razones, a la estrecha relación existente entre las propiedades cualitativas de los estados mentales y otro tipo de rasgos de los mismos con los cuales pudieran confundirse dichas propiedades, tales como su corporalidad y subjetividad. En segundo término, se necesita distinguir adecuadamente entre diferentes tipos de qualia. Mediante esta última operación podrá observarse que poco tienen que ver los qualia asociados a las emociones o el ánimo con las propiedades cualitativas que surgen asociadas a la percepción, de modo que no resulta pertinente considerar al conjunto completo de qualia como si fuese una totalidad homogénea. En lo que sigue, se examinan cada uno de estos aspectos por separado.

1. Las propiedades cualitativas de los qualia: recuperando el sentido común

\subsection{CoRPóREO NO ES LO MISMO QUE CUALITATIVO}

CUANDO HABLAMOS DE LOS QUALIA hacemos referencia a una amplísima gama de propiedades fenoménicas que acaecen como un flujo imparable vinculado a las sensaciones, emociones, estados de ánimo y otro tipo de estados mentales. 
Se trata de una dimensión inmanente de nuestra psique. No podemos dejar de experimentar qualia; ya sea que nos encontremos pensando, recordando, percibiendo, o en cualquier otro tipo de actividad mental, la experiencia cualitativa se encuentra siempre presente, más allá incluso de que seamos o no capaces de percatarnos de ella. Además, nos referimos a fenómenos que dadas sus propiedades intrínsecas no logran ser descritos acertadamente mediante las palabras. Podemos acercarnos a su caracterización a través del lenguaje verbal, haciendo referencia a los estados biológico-corporales asociados; sin embargo, aquello no constituye una descripción exacta ni completa de los mismos. Si digo, por ejemplo, que tengo algo de "tensión corporal", que "me encuentro inquieto" o que está activa mi ínsula anterior (intentando referirme con ello a mi experiencia interna), es evidente que fallo, ya que ninguno de estos términos precisa con fidelidad a qué quale específico me refiero. El componente interno, la fenomenología de los estados mentales, no resulta atrapable al hacer referencia a la dimensión corporal y fisiológica de estos. Existen variadas experiencias que pueden asociarse con los mismos estados biológico-corporales, de modo que este elemento, si bien es probable que constituya una condición necesaria para la existencia de cada quale en particular, no refiere con exactitud a su ontología cualitativa, el aspecto central de este tipo de fenómenos.

Con lo anterior no me refiero a la discusión sobre la posibilidad de reducción o no de los qualia a la física, sino al hecho de que, lingüística y comunicacionalmente, cuando hacemos referencia a través de las palabras al sustrato corporal de los qualia, no damos cuenta con ello, en términos referenciales, de su carácter cualitativo. Distinguir el carácter cualitativo de los estados mentales implica ir más allá del reconocimiento de estos como fenómenos biológicos y corporales. Cuando decimos "corporal", "encarnado" o "corporizado", no estamos dando cuenta con estos términos del carácter fenomenológico, experiencial y cualitativo de la vida mental, aun cuando es muy probable que este tipo de propiedades no puedan realizarse nunca sobre un substrato mecánico artificial.

\subsection{SubJETIVO NO ES EQUIVALENTE DE CUALITATIVO}

Por otra parte, el hecho de lue las propiedades cualitativas de los estados mentales no sean equivalentes a su carácter corpóreo es, tal vez, una 
de las razones por las cuales suele destacarse el carácter subjetivo de las mismas. Pareciera que la imposibilidad de 'atrapar' a través de las palabras el carácter cualitativo de los estados mentales, haciendo referencia a su vínculo corporal, llevara aparejada la necesidad de decir algo respecto de este tipo de propiedades que signifique un avance en la identificación de sus características fundamentales. En este contexto, cuando se dice que la conciencia es subjetiva, se hace referencia con ello a su ontología constituida desde un punto de vista personal exclusivamente (Humphrey, 1995; Searle, 1996). La dimensión cualitativa de la mente no es una entidad objetiva como ocurre, por ejemplo, con el comportamiento. No puede existir un quale fuera de la experiencia de un sujeto concreto, de modo que dichas propiedades no son observables 'desde fuera'. Se trata de una característica que distingue a los qualia de cualquier otro rasgo presente en la naturaleza y, como tal, constituye un enorme desafío para su investigación científica -una tradición de conocimiento que opera teniendo como principio fundamental la objetividad de lo estudiado-. A diferencia de lo que ocurre con el resto de nuestro mundo físico y cultural, respecto de las propiedades cualitativas de la mente, tan solo tenemos acceso a la propia. Si bien suponemos que nuestros interlocutores tienen expectativas, estados de ánimo, sensaciones y otros estados de características similares, la verdad es que solo somos testigos de nuestra propia mente como experiencia cualitativa y esta misma es también un fenómeno privado, inaccesible para los demás (Nagel, 2000).

Por otra parte, aun cuando la subjetividad es una propiedad que comparten todos los tipos de qualia existentes, no siempre nos percatamos de dicha condición. Así, por ejemplo, cuando tenemos rabia comprendemos sin grandes esfuerzos que los rasgos cualitativos de dicha experiencia son privados, más allá de que, por otra parte, podamos identificar las causas que lo provocan, así como sus rasgos expresivos asociados ${ }^{2}$. Sabemos que nuestro humor es un hecho que se

2 Usualmente se hace referencia a las emociones como estados multidimensionales (véase por ejemplo Aguado, 2005), identificándose un componente fisiológico, otro expresivo, otro subjetivo e, incluso, otro cognitivo de las mismas. Lo que aquí se sostiene es que el componente cualitativo de las emociones es el único que tiene un carácter subjetivo puesto que no puede accederse a este desde fuera, como sí ocurre respecto de los otros. Podemos registrar 'desde fuera' la fisiología de las emociones ajenas, observar el comportamiento que expresa quien tiene una emoción e, incluso, podemos conocer la dimensión representacional de la misma a través de su comunicación bajo un 
constituye en nuestra individualidad personal y al cual solo nosotros tenemos acceso; sin embargo, no ocurre lo mismo respecto de las sensaciones, aquellos estados cualitativos que participan en el proceso perceptual. Aun cuando se trata de estados producidos internamente por nuestro organismo, al igual que las variaciones del ánimo, solemos vivenciar los qualia perceptuales como características atribuibles al entorno y, por lo mismo, accesibles al punto de vista de cualquier observador. Las sensaciones son para nosotros eventos transparentes, de modo que frecuentemente no somos conscientes de los rasgos intrínsecos de dichas experiencias (Hartman, 1990). En este contexto, suponemos que los qualia perceptuales se configuran con unas características relativamente estándares para todos los seres humanos, asumiendo que tanto usted como yo, cuando vemos el cielo despejado, tenemos iguales experiencias internas de azul. Lo anterior ocurre puesto que en último término y de forma tácita atribuimos esta propiedad a una característica intrínseca del cielo y no a una construcción interna de nuestra mente. ¿Cuál es entonces la razón que explica el hecho de que para una variedad de qualia -las emociones- tengamos claridad respecto de su carácter subjetivo, es decir, sepamos a ciencia cierta que se trata de estados producidos por nosotros mismos, mientras que, en otros casos, correspondientes a qualia asociados a la percepción -las sensaciones-, tendamos a atribuir nuestro estado interno a las características del mundo exclusivamente, como si fuesen propiedades objetivas de este? Es probable que las diferencias en este sentido se relacionen con roles específicos que juegan uno y otro tipo de estados en el contexto del funcionamiento global de la mente, un problema que analizaré en detalle más adelante. Por ahora, baste con decir que referirnos al carácter privado o subjetivo de los qualia no es equivalente a identificar y describir sus propiedades cualitativas. Por ejemplo, Juan puede experimentar el frescor de la mañana mientras Pedro siente el calor de la calefacción al interior de su casa. Nosotros, observadores externos de dichas situaciones, podemos decir que ambos sujetos tienen experiencias

código lingüístico. Respecto del 'cómo se sienten' las emociones, nos encontramos con la imposibilidad de acceso a esta dimensión desde el punto de vista de la tercera persona. En este sentido, y siguiendo a Charland (2005), quien señala que es el carácter cualitativo de las emociones (su valencia) aquello que permite diferenciarlas de otros estados mentales, es que afirmo en este texto que las emociones son eventos privados. 
subjetivas, es decir, que nos resultan inaccesibles 'desde fuera'. ¿Qué hemos ganado en términos de identificar y describir las propiedades cualitativas de cada una de esas experiencias al referirnos a su carácter subjetivo? ¿Qué decimos respecto del 'cómo es ser' de la experiencia de frescor de Juan o del calor de Pedro, al señalar que se trata de estados subjetivos? Poco o nada. Diferentes personas pueden tener las mismas, o incluso diferentes, experiencias cualitativas y aquello no interfiere en el hecho de conservar la subjetividad personal de cada una. Subjetividad y cualidad no son la misma cosa, se trata de dos rasgos independientes del mismo fenómeno. Si queremos avanzar en la comprensión del rol que pudiera jugar el carácter cualitativo de los estados mentales, debemos intentar ir un poco más allá de la identificación de su vínculo con el cuerpo y su ontología subjetiva. En lo que sigue se intenta dar algunos pasos en dicha dirección.

\subsection{EL CARÁCTER CUALITATIVO DE LOS QUALIA}

De acuerdo con lo expuesto, el carácter cualitativo de los qualia no se subsume en su ontología encarnada y subjetiva. ¿A qué nos referimos entonces cuando decimos que un estado mental tiene características cualitativas? Se trata sin duda, de la propiedad más compleja de analizar de dichos estados. Podemos avanzar un poco en su comprensión si tomamos como ejes para el análisis dos rasgos principales que exhiben algunos fenómenos de este tipo. Se trata de dimensiones que aun cuando no nos permiten distinguir con completa exactitud entre dos estados particulares, sí posibilitan al menos su agrupación en clases de hechos distintos (Feldman, 2006). Me refiero, por un lado, a la valencia, es decir, el carácter agradable o desagradable de un quale determinado, y, por otra parte, al grado de activación o intensidad que este presenta. La rabia, el dolor, la ansiedad, el picor y la alegría tienen una valencia, en el sentido en que se vivencian internamente como situaciones placenteras o desagradables. Pero, además, cada uno de estos estados cualitativos exhibe también una cierta intensidad, puesto que puede variar dentro de un continuo de posibilidades de mayor o menor activación.

La consideración de ambos ejes -valencia y grado de activación- permite diferenciar distintas clases de fenomenología, de modo que, por ejemplo, la rabia y la alegría son dos tipos de estados claramente diferenciables en virtud de su 
valencia, esto es, el hecho de que se experimentan internamente como algo desagradable en el primer caso, y agradable en el segundo. Pero, además, al interior de una misma clase de valencia pueden identificarse diferentes subtipos de estados, según el grado de activación que cada quale presente. De esta forma, no es igual la sensación del dulzor de una manzana si se compara con la experiencia gustativa más intensa de la miel, del mismo modo que no es lo mismo experimentar una molestia producto de la falta de un lugar donde aparcar, que la ira que se siente al recibir un insulto en el trabajo.

La valencia y la intensidad de una experiencia son dimensiones que nos ayudan a identificar diferentes clases de qualia; sin embargo, las posibilidades de descripción que se realicen a partir de estos ejes tienen un límite, puesto que, finalmente, más allá de la clasificación que se realice a través de estas dimensiones, cada quale tiene además un carácter específico que no resulta descriptible mediante las palabras. Si bien existen estados que pueden presentar una misma valencia y ser similares en cuanto a sus grados de activación, como resultan ser, por ejemplo, el caso de la rabia y el miedo, subjetivamente nos percatamos de que entre estas dos emociones hay claras diferencias cualitativas y regularmente no nos confundimos entre tener una u otra experiencia. Sabemos cuándo estamos asustados y reconocemos también cuándo estamos enojados, del mismo modo que distinguimos el picor del dolor, el cansancio de la tristeza, o la angustia de la culpa. Se trata de tipos de experiencia diferentes, estados que se distinguen subjetiva y cualitativamente, a pesar de que no siempre seamos capaces de describir sus características específicas mediante términos lingüísticos. Las palabras no logran dar cuenta adecuadamente del carácter cualitativo de la experiencia puesto que la relación entre los términos lingüísticos y los estados mentales opera en un sentido inverso: las primeras adquieren su sentido a partir del carácter cualitativo de la experiencia y no viceversa (Bächler, 2006). ¿Cómo diríamos que algo es pesado, verde, duro o asqueroso si no fuese porque tenemos experiencias cualitativamente experimentadas en tales sentidos? La pesadez, el verdor, la dureza y la asquerosidad son propiedades que solo cobran sentido como adjetivos del mundo en tanto son vivenciados cualitativamente primero como experiencias subjetivas.

En este contexto, puede hipotetizarse que algunos qualia jueguen un rol en la construcción de significados y otro tipo de procesos cognitivos asociados. El 
caso de la experiencia de la rabia puede servir para ilustrar este punto. De acuerdo con Solomon (2007), el mayor exponente de las teorías evaluativas de la emoción, el enojo es una percepción implícita de injusticia, de tal forma que cuando nos sentimos molestos, estaríamos enjuiciando tácitamente una situación como inequitativa. Lo anterior, puesto que dicha experiencia, incluso antes de ser puesta en palabras, nos predispone cognitivamente en un cierto sentido. Versiones análogas podrían mantenerse de qualia como la nostalgia, que implicaría una apreciación de mejores escenarios en el pasado, o el dolor, que correspondería a una evaluación de daño en alguna parte de nuestro cuerpo.

Lo dicho en el párrafo anterior implica atribuir a los qualia, o al menos a algunos de sus tipos específicos, un carácter cognitivo. Sin embargo, comprender este carácter no es fácil, puesto que hacerlo implica traspasar los cánones de nuestra cultura dualista que separa a la mente del cuerpo, dejando fuera a la experiencia por considerarla un epifenómeno, y planteando, amparada en la metáfora computacional, que los únicos procesos que pueden considerarse de suyo como cognitivos son aquellos que tienen un carácter simbólico representacional (Bächler, 2014; Bem \& Keijzer, 1996). Mi impresión respecto de este último punto es que estamos confundiendo el resultado con el proceso. Como argumentaré en la sección 2.2, existen antecedentes suficientes para considerar la cognición como un proceso que transita desde propiedades cualitativas, subjetivamente experimentadas, hasta la realización de cadenas de símbolos representacionales susceptibles de ser manipulados a partir de reglas lógicas. Pero, además, para avanzar en la comprensión de esta idea, se requiere desagregar el conjunto amplio que llamamos qualia, diferenciando tipos y subtipos, con el objetivo de examinar, posteriormente, sus particulares roles en el contexto de la actividad global de la mente. Mediante esta última operación puede observarse que poco tienen que ver las propiedades cualitativas asociadas a estados, tales como las emociones o el ánimo, con las sensaciones de color u otras similares que surgen asociadas a la percepción, de modo que constituye un error ontológico tratar al conjunto fenomenológico completo como si fuese un bulto indiferenciado.

En lo que sigue intentaré avanzar en el sentido anterior, analizando dos diferentes clases de qualia y sus eventuales funciones en términos cognitivos. Para comenzar este examen, presentaré una perspectiva general acerca de la cognición, 
afirmando que conocer es modificar el formato que poseen los qualia. Se trataría de un proceso que, a grandes rasgos, consiste en ligar la fenomenología con los símbolos presentes en nuestra cultura, de modo que, bajo este enfoque, la experiencia puede ser concebida como el centro o punto de partida del conocer. No obstante, como argumentaré más adelante, parece ser que los qualia no siempre alcanzan a cumplir este rol. Existiría un tipo de qualia que, dadas sus características intrínsecas, no permitirían su procesamiento consciente y, en consecuencia, no cumplirían un papel central en la cognición. Dado su carácter encapsulado, estos estados actuarían de forma representacional en un sentido simple, es decir, re-presentando internamente la información del mundo sobre el cual se despliega la cognición. Para discutir esta posibilidad y evaluar el rol de las propiedades cualitativas de distintos tipos de estados, realizaré un experimento mental consistente en un intercambio de qualia emocionales, primero, y de qualia asociados a la percepción en segundo lugar ${ }^{3}$. Pretendo analizar en qué medida o forma la modificación del carácter cualitativo específico de una y otra variedad fenomenológica, conservando el resto de sus propiedades constantes, tiene consecuencias funcionales sobre los procesos cognitivos que se producen.

\section{El carácter cognitivo de la fenomenología}

EN LOS APARTADOS ANTERIORES he intentado destacar el carácter cualitativo de los estados mentales con el objetivo de sentar las bases que me permitan, ahora, evaluar la posibilidad de que esta característica juegue un rol específico en términos cognitivos. Como ya he señalado, la dimensión cualitativa de la mente es

3 Estos experimentos mentales guardan una estrecha semejanza con los casos de espectro invertido (Chalmers, 1999). Sin embargo, las situaciones que se plantean en este trabajo contienen algunas particularidades que permitirían analizar otros aspectos relacionados con el dilema de los qualia. En primer lugar, yo utilizo ejemplos de este tipo con el fin de examinar las relaciones existentes entre las propiedades cualitativas e intencionales de la mente, un objetivo diferente del que suele perseguirse cuando lo que se plantea son situaciones de espectro invertido, generalmente diseñadas para analizar la posibilidad de reducción de los qualia a su substrato físico. En segundo lugar, en este trabajo se discute la posibilidad de una modificación de experiencias emocionales, un ejemplo de quale que no suele ser considerado en la literatura filosófica relacionada. Por otra parte, respecto de los qualia perceptuales o sensaciones, aquí se considera un intercambio entre distintas modalidades sensoriales, de modo que sea posible observar un tipo de consecuencias distintas a las que sugiere la modificación de experiencias pertenecientes a una misma modalidad sensorial. 
una realidad inmanente: no podemos no experimentar qualia. Desde el mismo momento en el cual abrimos los ojos, despertando, por ejemplo, del sueño nocturno, comenzamos a sentir diferentes tipos de estados cualitativos y estos permanecen "hasta que nos dormimos de nuevo, o caemos en un estado comatoso, o nos morimos, o de uno u otro modo, quedamos inconscientes" (Searle, 2000, p. 19). Se trata de un flujo constante de sensaciones de todo tipo, emociones, sentimientos, dolores y un largo etcétera de estados y combinaciones de los mismos que se suceden sin parar, de tal forma que, al menos subjetivamente, tenemos la vivencia de que se trata de un continuo infinito de experiencia. Algún sentido adaptativo ha de tener este sinfín de estados mentales, por más que, históricamente, parte de la filosofía y la ciencia se haya empeñado en negar su importancia (Dennett, 1995; Churchland, 1981). A continuación, analizaré una situación hipotética con el objetivo de discutir la relevancia adaptativa que pudiera tener el carácter cualitativo de los qualia.

\subsection{UNA NEUROCIRUGÍA EMOCIONAL}

¿QUÉ PASARÍA SI PRODUCTO DE UNA NEUROCIRUgía se modificaran mis circuitos cerebrales de la rabia, conectándose a los de la pena, de modo que cada vez que me enfrente a situaciones que originalmente me causaban enojo, experimentase ahora una tristeza en el mismo grado? ¿Cambiarían de alguna forma mis estados conductuales y/o cognitivos como consecuencia de lo anterior ${ }^{34}$

4 El lector podría plantear que mi argumento presenta el siguiente problema: si se modifican los circuitos neuronales para invertir qualia emocionales, las consecuencias conductuales y cognitivas habrían de atribuirse a las modificaciones neuronales, antes que a la inversión cualitativa asociada. Luego, este escenario no probaría nada acerca del eventual papel cognitivo que jugarían dichos qualia. No me planteo este problema, puesto que, como argumentaré en la sección 2.2, pienso que existe una secuencia funcional que va desde lo cerebral a lo fenomenológico y desde ahí a lo cognitivo. Sin embargo, si el lector no comparte esta idea, me parece que es posible plantearse un experimento alternativo que de igual forma permite configurar la cuestión de fondo, esto es, la pregunta referida a si la inversión de qualia tiene consecuencias cognitivo-funcionales y cuáles serían. Pensemos en el caso de seres que viven en un mundo paralelo al nuestro donde todo se conserva constante, es decir, es exactamente igual a nuestro mundo, salvo que los seres que allí habitan tienen experiencias emocionales diferentes a las nuestras frente al mismo tipo de situaciones que nosotros vivimos en nuestro mundo. 
En principio, extrapolando escenarios a partir del recuerdo de mis experiencias previas con estas dos emociones, yo supongo que una situación como la descrita disminuiría mis posibilidades de adaptación al entorno. Probablemente, experimentando siempre tristeza en vez de rabia, sufriría constantes situaciones degradantes de forma innecesaria, ya que sería incapaz de reaccionar en el sentido de defenderme y modificar mi contexto cuando, por ejemplo, soy atacado verbalmente por un compañero de trabajo que me insulta. Resulta improbable que desde la pena experimentada yo exprese una respuesta del tipo: "ino voy a tolerar que me hables en ese tono!". Se trata de una expresión que no 'calza' con mi estado cualitativo actual de congoja, de modo que se vuelve una conducta improbable en este escenario. Pero más importante aún, para los efectos de este trabajo, los estados cognitivos que emergerían cada vez que soy agredido tendrían unas características diferentes a las que originalmente surgían en el contexto de la rabia experimentada. Tal vez mi experiencia cualitativa de tristeza dirija mi pensamiento en una dirección más reflexiva o introspectiva, y es probable que en este estado me sorprenda frecuentemente preguntándome cosas como "¿qué es aquello que tengo que hace que otros me humillen?”.

No parece entonces trivial un intercambio cualitativo de las características propuestas. Mientras que, anteriormente, la rabia experimentada me llevaba a defenderme y evaluar la injusticia de ciertas situaciones, la tristeza actual me deja sin los recursos necesarios para moverme de forma adaptativa en entornos agresivos. Considerándose el análisis de casos como este, puede observarse que el carácter cualitativo de los estados mentales es una propiedad en cierto modo cognitiva, puesto que 'abre' o 'cierra' determinadas posibilidades al pensamiento.

De hecho, se trata de un asunto que en cierto sentido ya ha sido tratado empíricamente en el ámbito de los estudios sobre las relaciones existentes entre la emoción y la cognición. En este plano, la investigación indica, por ejemplo, que la tristeza, una experiencia de valencia negativa, nos 'invita' a la reflexión, en la dirección de hacer introversión sobre nuestros pensamientos, valores y conductas (Stein \& Jewett, 1986). El carácter cualitativo de la pena, más allá de su valencia y grado de activación, es una experiencia que nos 'sugiere' que hay algo en nuestro interior que requiere ser atendido para su modificación o remoción. La rabia, en cambio, es una emoción que aparece relacionada en la literatura con la focalización en los obstáculos externos que impiden la consecución de un objetivo 
(Choliz, 2005), así como con la percepción de injusticia (Solomon, 2007). Se trata de un quale que, siendo también de valencia negativa como la tristeza, contiene una fenomenología intrínseca de otro orden, que 'promueve' la emergencia de estados cognitivos específicos. El enojo, a diferencia de la tristeza, dirige nuestra atención sobre el entorno indicándonos que algo va mal 'allá afuera', o que estamos siendo injustamente tratados, de modo que puedan plantearse acciones que corrijan la situación.

Como se desprende del análisis de este hipotético escenario, el aspecto intrínsecamente cualitativo de los qualia, al menos en el caso de las emociones, es una característica que tiene consecuencias funcionales en lo que respecta a los pensamientos que pueden surgir desde cada estado en particular. Dicho de otra forma, la propiedad cualitativa específica del quale sería, en último término, un estado cognitivo potencial o un significado en ciernes. En este contexto, cuando tenemos rabia, por ejemplo, estaríamos de algún modo 'pensando' que vivimos una situación injusta, aun cuando se trate de un proceso todavía implícito. Cada quale, más allá de ser placentero o desagradable, contiene en su interior elementos cognitivos específicos, una situación que, al ser reconocida, nos permite hablar de dichos estados como fenómenos, que son el centro de nuestra cognición, tal y como se describe a continuación.

\subsection{LOS QUALIA COMO CENTRO DE LA COGNICIÓN}

LO ANALIZADO EN LOS APARTADOS ANTERIORES permite argumentar que las propiedades cualitativas de algunos estados mentales, como los qualia emocionales, no son un 'capricho de la mente'. Se trata de propiedades que constituyen señales informativas o 'marcadores somáticos', que anuncian la llegada de cierto tipo de eventos (Bechara, Damasio, Damasio \& Anderson, 1994). Desde este punto de vista, los qualia pueden ser concebidos como cognitivos en un sentido amplio, puesto que proveen de información al organismo que los experimenta. Lo anterior requiere tener en cuenta una concepción de la cognición que vaya más allá de la noción tradicional como proceso explícito representacional, hasta una bajo la cual se reconozcan las capacidades sensitivas y adaptativas de la mente (Bem \& Keijzer, 1996). En ese contexto podrá comprenderse que cuando experimentamos las propiedades cualitativas de un estado mental; por ejemplo, antes 
de tomar una decisión, dichas propiedades son cognitivas puesto que anuncian posibles cursos de acción de los sucesos de una forma que es previa al proceso simbólico representacional que eventualmente pudiera realizarse. $\mathrm{Al}$ respecto, Pozo (2006), analizando las ideas de Damasio, señala:

Nuestro conocimiento tendría origen precisamente en esas representaciones encarnadas, surgiría de procesar en forma de emociones nuestras propias reacciones corporales ante los estímulos que nos afectan [...]. [T] oda nuestra actividad cognitiva está ligada a la presencia de marcadores somáticos, señales que nos informan de los estados favorables o desfavorables de nuestros órganos, de modo que podamos realizar acciones que nos ayuden a buscar lo que podíamos llamar, un "estado de fondo" del organismo, un equilibrio emocional basado en el alejamiento de los estados emocionales insatisfactorios y la consumación o acercamiento a los más agradables (p. 124).

La reflexión de Pozo sugiere que las emociones, más que ser meros acompañantes de los procesos cognitivos, serían el origen del conocer. Dicho de otra forma, la cognición, tal y como tradicionalmente la concebimos, sería el resultado del procesamiento de nuestras emociones. Es decir, antes del momento en el cual toman forma en nuestra mente las entidades representacionales que solemos caracterizar como cognitivas, habría propiedades cualitativamente experimentadas que son, por así decirlo, el primer estadio de nuestros procesos cognitivos. Además de la investigación llevada a cabo por Antonio Damasio, ya referida, existen estudios que dan cuenta de un adelantamiento por parte del cerebro a la toma de decisiones consciente (Bode et al., 2011). Dichos estudios son interpretados por Leys (2011) como una prueba de que la toma de decisiones no es un asunto del todo o nada. Con anterioridad al surgimiento de las representaciones mentales existen fenómenos de un orden distinto, los cuales constituyen, eventualmente, el 'material' sobre el cual se elaboran los estados intencionales.

¿Qué características tiene este material? Los estudios realizados por Damasio, así como la interpretación que de estos hace Pozo (2006), sugieren que es el carácter placentero o agradable de la emoción el elemento clave para la elaboración de representaciones mentales: "no hay cognición sin emoción [...], las emociones estarían ahí precisamente para marcar la valencia de esas representaciones, promoviendo acciones que las hagan más o menos probables" (p. 125). 
Se trata de una afirmación que entronca de muy buena forma con los hallazgos empíricos obtenidos por Lisa Feldman Barrett y su equipo de investigación en la Universidad de Boston. Este grupo, siguiendo un enfoque construccionista para el estudio de las emociones, identifica como el componente central de las mismas al "afecto nuclear" (core affect) que resulta de la combinación de la valencia de las emociones y el grado de activación o intensidad de las mismas (Barrett \& Russell, 2015). Se trata de 'fenomenología pura' o preintencional, es decir, que no se encuentra ligada a símbolos representacionales (Barrett, 2011). El ambiente y su permanente flujo de cambios son traducidos por el organismo a un código interno de variaciones fenomenológicas experimentadas privadamente. En un cierto sentido, esta fenomenología obedece a la resistencia que opone el organismo a los cambios con el objetivo de mantener su homeostasis (Barrett \& Russell, 2015). El afecto nuclear así entendido equivale a una forma de conocimiento que hace parte de nuestro 'cableado' de nacimiento. Se trata de un 'barómetro' de sentimientos que proveen al organismo de una medida para estimar los cambios que se producen en su ambiente externo, al interior del cual la valencia constituye su elemento clave, el 'calor' o el quale de las emociones (Charland, 2005).

En palabras de algunos de los máximos exponentes de la teoría, Barrett, Mesquita, Ochsner \& Gross (2007): "el afecto nuclear no es en sí y por sí mismo suficiente para la representación mental de la emoción. La emoción es un estado mental intencional, un estado afectivo que es acerca de algo" (p. 379). El paso desde el afecto nuclear hasta las emociones como estados representacionales requiere procesar cognitivamente la experiencia afectiva a través de su valorización y categorización: "ambos procesos, la valorización y la categorización, modifican el estado de la persona creando un producto emergente que es a la vez afectivo y conceptual" (Barrett, 2006, p. 36). A través de distinciones cognitivas, preferentemente lingüísticas, nuestra experiencia emocional básica es ligada a determinados conceptos que permiten su organización otorgándole un carácter intencional que la 'dirige sobre' o 'refiere a' algo distinto de ella misma. Podríamos decir que, a través de este proceso de categorización, la emoción se 'expande' o 'conecta' con determinados objetos del mundo interno o externo de quien la experimenta. 
Este 're-formatear' los estados cualitativos permitiría refinar la experiencia aun cuando la representación resultante no sea tampoco un estado puramente simbólico, como han expresado algunos cognitivistas clásicos como Fodor (2003). Por el contrario, las nuevas representaciones mentales que se alcanzan, más allá de su carácter simbólico, llevarían en su interior el 'germen' de lo cualitativo original, una situación que de ser estudiada en profundidad debería contribuir a explicar el carácter indisociable de las propiedades intencionales o representacionales y la fenomenología de los estados mentales que algunos autores refieren (Horgan \& Tienson, 2002). Esta doble dimensión, intencional-cualitativa, es una característica que poseen la gran mayoría (si no todos) los estados mentales, aun cuando en el producto final de aquello que denominamos pensar, aprender, o emocionarnos sea difícil de observar (Duncan \& Barrett, 2007).

No obstante, hay varias razones por las cuales resulta sumamente complejo observar este modo de funcionamiento de la mente. En primer lugar, como ya he señalado, en el continuo de la experiencia las propiedades cualitativas y representacionales de los estados mentales se vivencian como un todo unitario, de modo que aun cuando nosotros separamos para su análisis ambos dominios de lo mental, en verdad, aquello es indivisible en términos experienciales.

Por otra parte, existen algunas dificultades de orden más concreto que complican la comprensión de las relaciones existentes entre ambos dominios del funcionamiento mental. Estas se derivan del hecho de que los qualia no siempre se encuentran disponibles para su procesamiento consciente, una situación que obedece a dos razones principalmente. Por un lado, debido al complejo funcionamiento de la mente que implica la combinación y superposición de diferentes estados cualitativos en forma simultánea, existen situaciones bajo las cuales las propiedades cualitativas de las emociones $\mathrm{y} / \mathrm{u}$ otros estados similares no ingresan al campo del darse cuenta -awareness-, permaneciendo como telón de fondo o contexto de nuestros procesos cognitivos. Por otra parte, parece ser que algunos qualia perceptuales o sensaciones se encontrarían encapsulados debido a la deriva filogenética de la cual provienen, de tal forma que resultan impenetrables para su procesamiento consciente, tal y como se analiza a continuación. 
3. Fenomenología no cognitiva: los qualia encapsulados e intercambiables

Como adelanté en la primera parte de este trabajo, no toda la fenomenología humana jugaría un rol en los términos señalados. Concretamente, hay algunos casos correspondientes a las sensaciones o qualia perceptuales en los que el carácter cualitativo no parece desempeñar un rol relevante en términos cognitivos. Por ejemplo, ¿cuál es la razón por la que el color verde se experimenta con su verdor característico o el sabor dulce con su conocido dulzor? ¿Qué pasaría si cada vez que nos enfrentamos a la percepción del cielo, en vez de constituirse una sensación azulada en el espacio mental interno, ocurriera que experimentamos rojez u otro tipo de experiencia de color? Las preguntas anteriores hacen referencia al problema de identificar el rol que tiene el carácter cualitativo de las sensaciones sobre los procesos cognitivos. ¿Variaría nuestro pensamiento en algún sentido si nuestros qualia perceptivos se viesen alterados, de un modo similar al cual variarían si intercambiásemos nuestras emociones, como en el experimento de la neurocirugía emocional propuesto?

Hablamos de la posibilidad de qualia perceptuales intercambiables, una situación que en verdad ya existe, desde la década de los años sesenta del siglo XX, época durante la cual el investigador norteamericano Paul Bach-y-Rita inauguró un campo de investigación denominado substitución sensorial, consistente en el reemplazo de qualia perceptuales. El estudio permitió a una persona ciega de nacimiento experimentar representaciones del espacio reemplazándose una entrada sensorial visual por otra de tipo táctil (Bach-y-Rita et al., 1969). Este experimento, desarrollado al alero de la Universidad Nacional Autónoma de México, constituye el pilar fundamental de un ámbito de investigación en neuro-plasticidad y rehabilitación para personas con discapacidad.

No obstante el interés práctico que este campo de investigación presenta, los casos de substitución sensorial no nos permiten realizar un análisis riguroso del carácter eventualmente cognitivo de la fenomenología sensorial. Lo anterior, debido al hecho de que el reemplazo que se realiza en estos casos no constituye un ejemplo de intercambiabilidad exacta ni completa entre una y otra modalidad de qualia perceptuales. Para evaluar el carácter cognitivo o no de un tipo de sensación, se requiere contar con una situación de total intercambiabilidad de qualia 
entre dos tipos de experiencias sensoriales, pero que conserve, no obstante, el mismo espectro de variabilidad posible entre una y otra modalidad.

Imaginemos, como ejemplo, que toda la gama de experiencias asociadas a la percepción del color se reemplaza por sensaciones de tipo auditivo. Consideremos, además, que en el intercambio se mantiene el mismo espectro de variabilidad en relación con los límites de lo perceptivamente posible, así como respecto de los márgenes de modificación existentes entre una experiencia y otra. ¿Tendría una situación de tales características alguna consecuencia en términos funcionales, en el sentido de transformar los estados mentales globales que se produzcan? Examinamos a continuación un caso que sí parece cumplir con los requisitos especificados para evaluar este punto.

\subsection{NeIL HARBISSON: LA ACROMATOPSIA DE MARÍA REVISITADA}

Neil Harbisson es Un aRTista visual británico-español portador de una rara mutación genética, causante de una condición visual denominada acromatopsia o ceguera de colores. Esta característica le significa a Harbisson, de forma similar al caso de María, la neurocientífica aislada (Jackson, 1986) ${ }^{5}$, una incapacidad para 'ver' colores. De esta forma, su vida mental internamente experimentada se compone únicamente de tonalidades de grises, claros y oscuros. Así, por ejemplo, cuando Neil camina por el parque de la Ciudadela ${ }^{6}$ un día de otoño, no puede experimentar la maravilla del paisaje que configuran las hojas de color café, cayendo sobre el verde de la laguna artificial, rodeada de patos y botes de colores. Sin embargo, a diferencia de María, Harbisson, quien es considerado el

5 El caso señalado corresponde a un experimento mental propuesto por el filósofo australiano Frank Jackson. María es una investigadora que posee todo el saber neurológico respecto del funcionamiento del cerebro, relativo al procesamiento del color. Sin embargo, esta científica nunca ha salido de su habitación, dentro de la cual, ve solo en blanco y negro. Producto de lo anterior, ella se ve imposibilitada para tener acceso a la experiencia consciente del color.

6 Zona verde ubicada en Barcelona y que es uno de los lugares más significativos de la capital de Cataluña, lugar donde vive Neil Harbisson. 
primer ciborg ${ }^{7}$ reconocido oficialmente por el estado inglés, sí tiene la posibilidad de diferenciar entre el verde y el café, aun cuando no tenga subjetiva, corporal y cualitativamente la experiencia cromática. Lo anterior ocurre gracias a que este sujeto ha incorporado a su cuerpo una prótesis que le permite identificar la presencia de cada uno de los colores y tonos que nosotros reconocemos como parte de nuestro mundo de experiencias cromáticas, solo que en el caso de Harbisson esto sucede bajo un formato sonoro. La prótesis en cuestión, denominada eyeborg, detecta las variaciones de luminosidad en el ambiente traduciendo la información a un formato sonoro, que Neil percibe a través del canal auditivo. De esta forma, cuando él pasea por el parque de la Ciudadela, es igualmente impactado por la información que proporcionan los colores del paisaje, solo que no experimenta aquello como experiencias de color, sino de sonido. De hecho, Harbisson, como artista que es, se ha dedicado a la tarea de viajar por distintas ciudades del mundo 'escuchando' los colores que predominan en cada una de estas. Así, según su experiencia, Mónaco suena azul y salmón rosado, mientras que en Lisboa predominan el amarillo y el turquesa.

La situación de Neil Harbisson, constituye el mejor ejemplo vivo de intercambio de sensaciones, puesto que se trata de un caso que conserva la misma gama de variabilidad cualitativa posible para uno y otro tipo de sensación visual o sonora. En este caso hay una correspondencia exacta entre las posibilidades de variación visual de los colores y los modos de experimentación de los sonidos. A cada color le corresponde un sonido específico. En palabras del mismo Harbisson, entrevistado por el escritor español Juan José Millás:

-El color y el sonido -dice- poseen una cosa en común: que los dos tienen frecuencia. La frecuencia de cada color se corresponde con una nota musical que no podemos escuchar con el oído porque es excesivamente aguda y porque es una onda de luz y no de sonido. Lo que yo hago es una transposición de las frecuencias de luz o de los colores a frecuencias de sonido.

- ¿La relación entre los colores que miras y los sonidos que escuchas no es, entonces, arbitraria?

7 Organismo compuesto de elementos orgánicos y dispositivos cibernéticos mecánicos, generalmente con la intención de mejorar las capacidades de la parte orgánica mediante el uso de tecnología. 
-En absoluto. Si el oído humano pudiera escuchar la frecuencia del color rojo, escucharíamos la nota fa, aproximadamente (Millás, 2012).

Se trata de un intercambio de la experiencia perceptual visual del color por sonido, conservando constante el espectro de variabilidad posible, pero modificándose el carácter cualitativo de la sensación experimentada. La pregunta que naturalmente surge, en el contexto de este trabajo, es por las eventuales consecuencias funcionales que esta situación tiene. ¿Se modifican en algún sentido los estados cognitivos que se producen en el contexto de un intercambio de qualia sensoriales como el descrito?

En el caso específico de Neil Harbisson no podemos dar una respuesta que resulte válida, puesto que se trata de una persona que nació con acromatopsia, de modo que no hay un antes y un después que nos permitan hacer el contraste. Sin embargo, lo que sí sabemos es que en su situación existían dificultades para moverse adaptativamente con anterioridad a la instalación del eyeborg:

- ¿Pero ver en blanco y negro no implica también alguna dificultad de orden práctico?

-Alguna, sí. Con los grifos, por ejemplo, porque no siempre el del agua caliente está a la izquierda. O con los cargadores de baterías, en los que la luz verde indica una cosa y la roja otra. Los mapas son un caos total. También hay muchos trabajos a los que no se puede acceder viendo en blanco y negro (Millás, 2012).

Sin embargo, una vez realizada la instalación del dispositivo comentado, su capacidad de movimiento resulta totalmente adaptativa, como si experimentase los colores en su sentido cualitativo. Más aún, ahora Harbisson puede detectar la presencia de colores que el ojo humano no percibe, como los infrarrojos o los ultravioleta, mediante su asociación con sonidos efectivamente audibles.

Este caso muestra que, a diferencia de las emociones, en al menos algunos de los denominados qualia perceptuales podemos hablar de 'fenomenología no cognitiva'. En el caso del color, el carácter cualitativo de la experiencia no resulta relevante en términos funcionales, su percepción no indica nada por sí mismo. Las sensaciones de rojo, de verde, o de azul, internamente experimentadas, se relacionan representacionalmente con diferencias en el mundo allá afuera, pero sus propiedades cualitativas no tienen consecuencias 
funcionales en un sentido cognitivo. El quale del azul resulta intercambiable por uno de otro tipo -como de hecho ocurre en el caso del daltonismo-, y nada relevante en términos cognitivos ocurre. El valor cognitivo de la percepción del color no se encuentra en sus características cualitativas intrínsecas, sino más bien en lo que ellas representan, a saber, variaciones cuantitativas de luz en el ambiente.

Por otra parte, como ya señalé en apartados anteriores, el carácter no cognitivo de este tipo de fenomenología se relaciona directamente con la imposibilidad de acceso autoconsciente que tienen las sensaciones. A diferencia del caso de las emociones, nosotros no solemos ser conscientes de la subjetividad de las sensaciones. Sus características cualitativas específicas son experimentadas, en general, como rasgos extrínsecos, dependientes de los objetos del mundo más allá del espacio intracraneal. Por ejemplo, la sensación de blanco y la percepción del mismo van indisolublemente atadas en la experiencia, de tal forma que fenomenológicamente, cuando yo percibo la pared de mi casa, no diferencio entre mi alba sensación aquí adentro y las características constitutivas de la pared allá afuera. En condiciones regulares, los seres humanos no nos damos cuenta de que cuando percibimos realizamos una construcción mental interna llamada sensación. La anterior es una situación especialmente notoria en el caso de la percepción visual (Humphrey, 1995). Los qualia visuales son estados encapsulados, en el sentido fodoriano del término (Fodor, 1986), y en contextos normales resultan impenetrables para la conciencia. A diferencia de las emociones, en este caso ${ }^{8}$, el impacto se observa directamente sobre nuestro comportamiento, sin pasar por el filtro de la conciencia, en el sentido de percatación. Se trata de una situación ventajosa en términos de la rapidez y economía energética que supone. ¿Implicaría alguna mejora adaptativa la toma de consciencia de las características intrínsecas de los qualia perceptuales? ¿Qué pasaría si experimentásemos las sensaciones asociadas a la percepción de las veredas, personas, etc., como asuntos internamente

8 La capacidad de darnos cuenta del carácter subjetivo de las sensaciones es una propiedad que se presenta en diferentes grados en cada una de las distintas modalidades sensoriales. Estados como los del tacto y el gusto se encuentran asociados a una mayor percatación del carácter subjetivo de dichas experiencias, si se comparan con lo que ocurre en el caso de la percepción visual o auditiva, un tipo de sensaciones donde prácticamente todo lo que se experimenta suele ser atribuido a las características externas del estímulo. 
construidos? ¿Es verdad que allá adentro está mi hijo jugando en su habitación o se trata tan solo de una proyección de mi mente? En principio, parece ser que gracias a que confiamos en lo que vemos, nos movemos adaptativa y fluidamente por el mundo. Respondemos a quien nos habla, nos desplazamos libremente por donde no se sienten obstáculos, y nos detenemos frente a la percepción de los objetos sólidos en el camino. En este contexto, dudar de lo que vemos podría ser un riesgo, sobre todo en aquellos escenarios donde la demora en la toma de decisiones significa la diferencia entre la vida y la muerte. Piense usted en los esquimales y su percepción de las variaciones de tonos de blanco en los hielos, un tipo de sensación que se asocia a la discriminación entre un piso firme y caminable, o el peligro de caer en aguas gélidas. En situaciones como estas, la inmediatez de las conductas y su determinación por experiencias perceptuales implícitas constituyen sin duda una ventaja adaptativa y, en algunos casos, la diferencia entre la vida y la muerte. Sin embargo, una situación diferente es la que ocurre en relación con algunos de nuestros entornos sociales y culturales, donde la duda respecto de las propias percepciones podría llegar a configurar una ventaja que facilitase la interacción social, aportando frescura y flexibilidad al comportamiento. Por ejemplo, qué distintas serían nuestras aulas escolares si los docentes asumieran que el aprendizaje es un proceso subjetivo y cuestionaran la posibilidad que tienen sus alumnos de percibir literalmente lo que se verbaliza cuando se enseña. Sin embargo, la investigación referida a las concepciones que los docentes tienen sobre el aprendizaje muestra que la asunción de perspectivas constructivistas es la excepción y no la regla en el mundo educativo (Pozo et al., 2006).

No obstante, es probable que la especie humana vaya camino de un cambio filogenético en el sentido descrito, una situación que pudiera verse impulsada por las notables modificaciones que experimentan nuestros contextos sociales y culturales. En nuestro mundo, cada vez se vuelven más importantes las interacciones humanas, predominan las actividades mentales por sobre las físicas y las actividades cotidianas se ven acompañadas además de una progresiva importancia de los contextos virtuales (Carr, 2011). Tal vez, la presencia de estos nuevos entornos se traduzca en el largo plazo en un nuevo tipo de mentalidad humana, donde la conciencia de la subjetividad de la mente cuando percibe sea la norma y no la excepción. Puedo observar en primera persona esta tendencia, cuando me recuerdo a mí mismo diciéndole a mi hijo una frase que ya se ha vuelto 
costumbre en mi hogar: "ino-me-gusta-que-juegues-tanto-a-ese-juego-de-matar-gente!”. Y mi hijo por su parte respondiéndome -también como siempre-: “ies-un-juego-papá, -no-es-la realidad-!”.

\section{Conclusiones y proyecciones}

EN ESTE TRABAJO HE PRESENTADO ALGUNAS REFLEXIONES referidas al rol que podría caberle a la dimensión cualitativa de la mente en los procesos cognitivos. Al hacerlo he adoptado implícitamente una perspectiva evolucionista, asumiendo que dichos estados son el resultado de una selección filogenética. Por otra parte, la propuesta asume como punto de partida la existencia de los qualia, sin entrar en el llamado problema duro o difícil de la conciencia, referido a la identificación de los mecanismos por los cuales se produciría la experiencia (Chalmers, 1999). Siguiendo este razonamiento, puede decirse que el trabajo presentado apunta a resolver una de las cuestiones que caen dentro de la categoría que el filósofo australiano denomina problemas 'fáciles' de la conciencia. Intentar resolver este tipo de asuntos podría ser parte de una estrategia inductiva, que tal vez tenga algunos 'coletazos' sobre el problema difícil. El primero de ellos sería la conveniencia de desagregar el conjunto que en forma indiferenciada llamamos qualia y comenzar un estudio distinguiendo tipos y subtipos, tal y como he avanzado en esta propuesta.

En segundo lugar, el trabajo desarrollado sugiere la conveniencia de revisar algunos conceptos relacionados con la perspectiva denominada mente corporalizada (embodied mind), conectando de mejor forma el estudio fenomenológico con este enfoque, dos perspectivas que luego de la muerte de Francisco Varela y la suspensión de su neurofenomenología ${ }^{9}$ han quedado escindidas. Una adecuada

9 Mediante este proyecto, el científico chileno ya fallecido pretendía avanzar en el conocimiento de la conciencia, utilizando distinciones experienciales provenientes de la fenomenología de MerleauPonty y correlacionando a su vez estos estados y sus propiedades con el funcionamiento de su substrato cerebral. Para cumplir con este propósito, el proyecto de investigación utilizaba sujetos expertos en meditación budista, una técnica que permite un exhaustivo grado de focalización en diferentes tipos de estados de conciencia. Para una revisión de los detalles referidos al proyecto neurofenomenológico, recomendamos revisar Rudrauf, Lutz, Cosmelli, Lachaux \& Le van Quyen, 2003. Para un análisis crítico de la continuidad de dicho proyecto en el presente, puede verse Bächler, Poblete \& Poblete, 2012. 
integración de ambas perspectivas permitiría reformular el concepto de cuerpo presente en la perspectiva corporalizada, incorporando de mejor forma la dimensión sintiente o cualitativa de la mente en el embodied, un concepto que por ahora acentúa más los aspectos sensorio-motrices y biológicos del cuerpo que su dimensión experiencial cualitativa.

Finalmente, quedan como asuntos pendientes las posibles investigaciones empíricas que pudieran derivarse de este trabajo, el cual es, por ahora, sólo una especulación razonada, sobre un tema difícil, y que requiere por cierto de evidencia empírica para poder sustentarse adecuadamente.

\section{Referencias}

Aguado, L. (2005). Emoción, afecto y motivación. Madrid: Alianza.

Bächler, R. (2006). Conciencia y lenguaje: análisis del vínculo proyectado a través de la intencionalidad. Gaceta de Psiquiatría Universitaria, 2(4), 432438.

Bächler, R., \& Poblete, O. (2012). Interacción, emoción y cognición: Una aproximación integrada a la comprensión del comportamiento humano. Anales de Psicología, 28(2), 490-504.

Bach-y-Rita, et al. (1969). Vision Substitution by Tactile Image Projection. Nature, (221), 963-964.

Barret, L. F., \& Russell, J. (2015). The Psychological Contruction of Emotion. London: Guilford Press.

Barrett, L. F. (2011). Constructing emotion. Psychological Topics, 3(20), 359380.

Barrett, L. (2006). Solving the Emotion Paradox: Categorization and the Experience of Emotion. Personality and Social Psychology Review, 10(1), 20-46.

Barrett, L. F., Mesquita, B., Ochsner, K., \& Gross, J. (2007). The Experience of Emotion. Annual Review of Psychology, 56, 373-403.

Bechara, A., Damasio, A. R., Damasio, H., \& Anderson, S. (1994). Insensitivity to Future Consequences Following Damage to Human Prefrontal Cortex. Cognition, 50, 7-15.

Bem, S., \& Keijzer, F. (1996). Recent Changes in the Concept of Cognition. Theory \& Psychology, 6(3), 449-469. 
Bode, S. et al. (2011). Tracking the Unconscious Generation of Free Decisions Using UItra-High Field Fmri. PLoS ONE, 6(6), e21612.

Carr, N. (2011). ¿Que está haciendo internet con nuestras mentes superficiales? Madrid: Santillana.

Chalmers, D. (1999). La mente consciente. En busca de una teoría fundamental. Barcelona: Gedisa.

Chalmers, D. J. (2010). The Character of Consciousness. Oxford: Oxford University Press.

Charland, L. (2005). The Heat of Emotion. Valence and the Demarcation Problem. Journal of Consciousness Studies, 12(8-10), 82-102.

Choliz, M. (2005). Psicología de la emoción: el proceso emocional. Recuperado de www.uv.es/=choliz

Churchland, P. (1981). Eliminative Materialism and Propositional Attitudes. The Journal of Philosophy, 78(2), 67-90.

DeLancey, C. (1996). Emotion and the Function of Consciousness. Journal of Consciousness Studies, 3(5-6), 492-499.

Dennett, D. (1995). La conciencia explicada: una teoria interdisciplinar. Madrid: Paidós.

Duncan, S. \& Barrett, L. F. (2007). Affect is a Form of Cognition: a Neurobiological Analysis. Cognition \& Emotion, 28, 1184-1211.

Feldman, L. (2006). Valence is a Basic Building Block of Emotional Life. Journal of Research in Personality, 40(1), 35-55.

Flanagan, O. (1991). The Science of the Mind. Cambridge, MA: MIT Press.

Fodor, J. (1986). La modularidad de la mente. Madrid: Morata.

Fodor, J. (2003). La mente no funciona así. Madrid: Siglo XXI.

Hartman, G. (1990). The Intrinsic Quality of Experiencie. Philosophical Perspectives, 4, 31-52.

Horgan, T., \& Tienson, J. (2002). The Intentionality of Phenomenology and the Phenomenology of Intentionality. En: D. J. Chalmers. (Ed.). Philosophy of Mind: Classical and Contemporary Readings (pp. 520-533). Oxford: Oxford University Press.

Humphrey, N. (1995). Una historia de la mente. La evolución y el nacimiento de la conciencia. Barcelona: Gedisa. 
Jackson, F. (1986). What Mary Didn't Know. The Journal of Philosophy, 83(5), 291-295.

Leys, R. (2011). The Turn to Affect: A Critique. University of Chicago Press, 37(3), 434-472.

McGinn, C. (1989). Can We Solve the Mind-Body Problem? Mind, (98), 349366.

Millás, J. J. (2012, enero 17). El ciborg del tercer ojo. El País. Recuperado de http://tecnologia.elpais.com/tecnologia/2012/01/17/actualidad/13268 03391_782100.html

Nagel, T. (1974). What is Like to Be a Bat. Philosophical Review, 83(4), 435-450. Nagel, T. (2000). Otras mentes. Barcelona: Gedisa.

Pozo, J. I. (2006). Adquisición de conocimiento. Madrid: Morata.

Pozo, J. I. et al. (2006). Nuevas formas de pensar la enseñanza y el aprendizaje. Las concepciones de profesores y alumnos. Barcelona: Graó.

Searle, J. (1996). El redescubrimiento de la mente. Barcelona: Grijalbo Mondadori.

Searle, J. (2000). El misterio de la conciencia. Barcelona: Paidós.

Solomon, R. (2007). Ética emocional: una teoría de los sentimientos. Barcelona: Paidós.

Stein, N. L., \& Jewett, J. L. (1986). A Conceptual Analysis of the Meaning of Negative Emotions: Implications for a Theory of Development. En: C. E. Izard \& P. B. Read. (Eds.). Measuring Emotions in Infants and Children (Vol. 2). Cambridge Studies in Social and Emotional Development (pp. 238-267). New York: Cambridge University Press.

Varela, F. (2000). El fenómeno de la vida. Santiago de Chile: Dolmen. 